\title{
GUI based Wireless Communication for Plant Automation
}

\author{
K. Vidyasagar \\ Department of Electronics and \\ Communication Engineering \\ SSIT, B-Gangaram, Sattupalli
}

\author{
T. Chaitanya \\ Department of Electronics and \\ Communication Engineering \\ SSIT, B-Gangaram, Sattupalli
}

\author{
D. Swapna \\ Department of Electronics and \\ Communication Engineering \\ SSIT, B-Gangaram, Sattupalli
}

\begin{abstract}
The field instruments of the process plant are communicated with the remote control room where the supervisor is alarmed to monitor the process parameters regulating at some desired value. The onsite monitoring is limited to only specific supervisors. This paper aims to interface the plant parameters to web server for constant monitoring the state of the process plant at various locations by multiple supervisors. This paper considered the temperature parameter $(\mathrm{PV})$ to regulate at some desired value. The measured signal is logged by the arduino board and then the signal is transferred to the AT mega 328 microcontroller. PID algorithm is applied to regulate the parameters. AT mega 328 is used as a controller and to output the data serially to IEEE 802.4.15 wireless communication device. The data received at the personal computer for analysis and monitoring the state of the plant parameters using Graphic user interface (GUI) approach. GUI is implemented with the support of python, internet of things application. The developed model produced more reliable results to consider for implementation in process plants.
\end{abstract}

\section{Keywords}

Process temperature, Arduino board, AT mega 328 microcontroller, Graphic user interface.

\section{INTRODUCTION}

Automation of the process parameters with programmable logic controllers (PLCs) with applied Supervision and data acquisition system (SCADA) or with Distributed control system (DCS) are having significant role in automation. With rapid advancement of technology the art of supervision of the process plant parameters on the off site is having a significant scope of research in plant automation.By adjusting the gate valve the flow rate of the fluid is maintained to be constant in order to avoid frictional forces of the fluid. An Arduino board is used to read the process temperature value and the flow rate of the fluid. Inbuilt 10 bit resolution ADC converts the input analog signal into digital signal. Atmega 328 microcontroller is programmed to process the received input signal to generate the error signal for enabling the actuating elements. Proportional ( p ) + integral ( I ) + Derivative (D) controller is applied to dominate on the offset and to improve the response of the system by adjusting the gain of the controller. The controller is tuned using Nichols Gigglers method. The error signal of the controller causes to drive the final controller i.e. Triac. The output signal of the microcontroller is converted into serial form using Max 232 Then the data is transmitted using IEEE 802.4.15 wireless communication protocol. The receiver is interfaced to the web server. Python software is used to plot the response of the process parameter w.r.t to the received input data. An open source of internet of things application is used to store and receive data from things with the support of HTTP protocol over the internet.

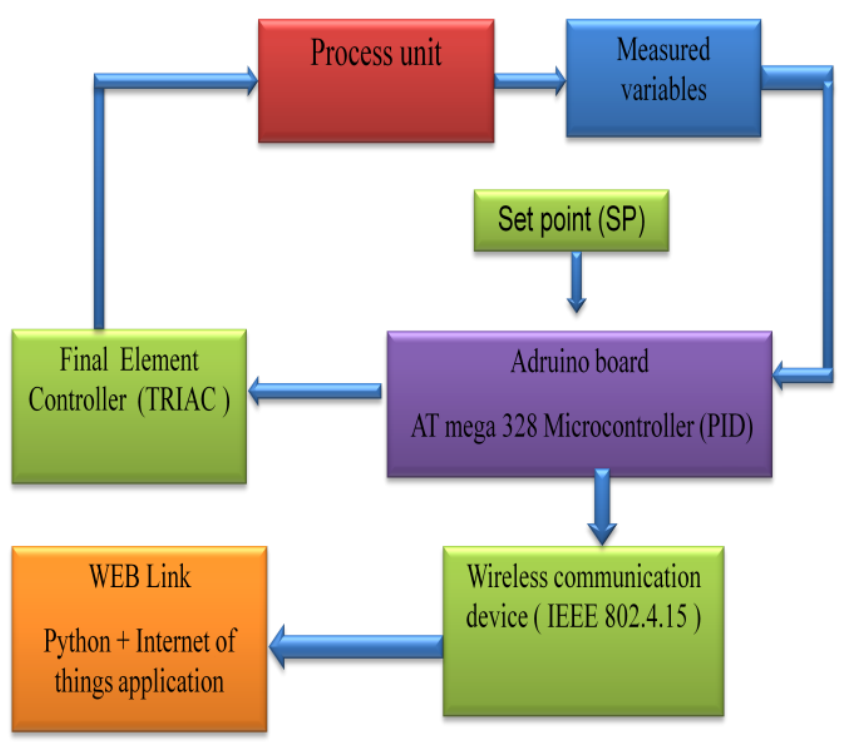

Fig1.1 Blog diagram of the proposed model

\section{LITERATURE SURVEY}

This paper proposed new software called GUI based real time industrial automation Software. This has the ability to extract the run time data from the server for monitoring the industry [1].

This paper proposed internet linked plant automation. The plant is to be supervised remotely to prevent the downtime and maximizing the operational life of the plant [2]

This paper discusses a simulation software package for the grain silo of Jordan. The author discussed the anticipated benefits and various phases of implementation. This paper considered the PLC and operator interface for monitoring the plant parameters [3]

This paper proposed a console master computer (CMC) to monitor various physical nodes. The proposed method is able to analyze the content area network and data traffic management [4].

This paper proposed a novel particle swam optimization mechanism to optimize the plant variables. The author applied the proposed algorithm for natural gas liquefaction plant for testing the efficiency and reliability [5] . 
This paper describes the significance of ZIG BEE technology for automation. The author focused on various communication methodologies and compared with Zig bee technology for automation of the devices [6].

This paper describes remote controlling of the crane using mobile phone. This describes the communication methodology between the Supervisory control and data acquisition system (SCADA) server, general packet radio service (GPRS) and wireless application protocol (WAP) [7] .

\section{HARDWARE IMPLEMENTATION}

This paper considered two process parameters. The fluid temperature is measured using immiscible thermostat type temperature sensor. The sensor is mounted in the pipe line. The flow rate of the fluid in the pipeline is fixed to a constant value. So that the frictional forces on the influence of the fluid is restricted. The fluid temperature is to be regulated using the controlling unit. The control unit is designed using arduino board to sense the field instrument signals and to drive the output variables. An AT mega 328 microcontroller is inbuilt within the board to control the process parameter and to transmit the data serially. PID controller concept is imposed in the microcontroller.

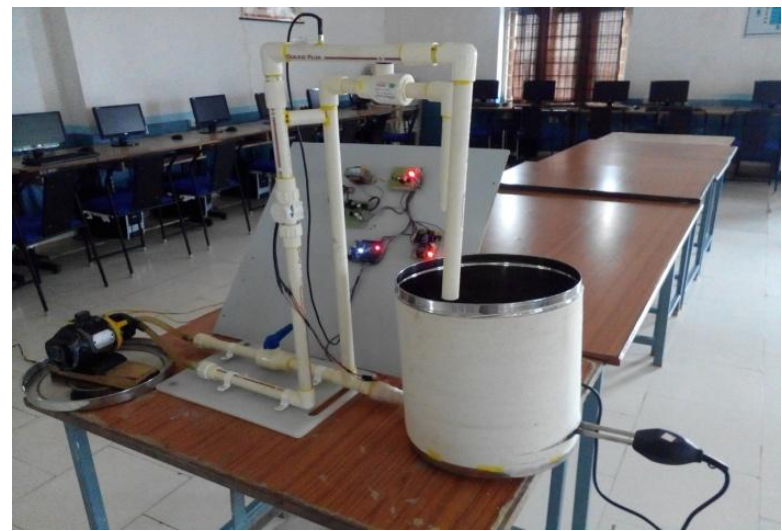

Fig 3.1 Experimental setup, control and transmitter section

The microcontroller behaves as a soft PID controller. The measured signal is compared with the desired signal cause to generate the error signal. This error signal is used to fire the triac to deliver the load to the heating element. The measured signal and the error signal from the controller are transmitted serially to the control room. IEEE 802.15.4 wireless communication device is used to transmit and to receive the signal serially.

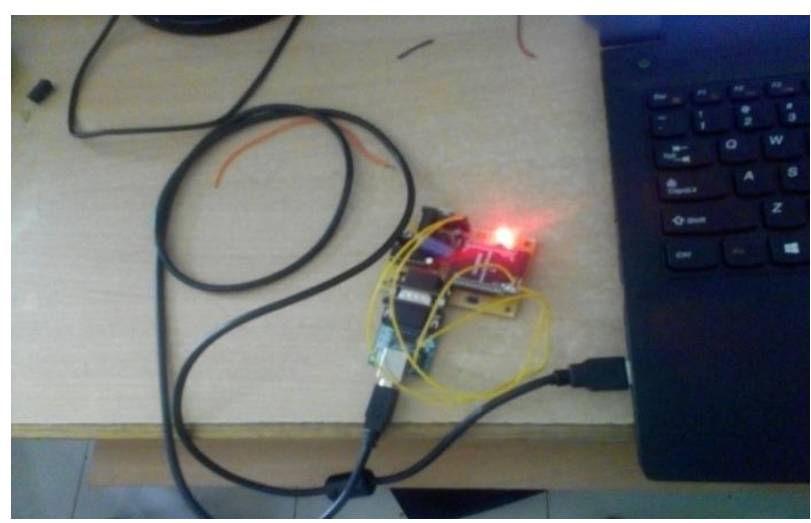

Fig 3.2 Receiver section at the personal computer

\section{SOFTWARE IMPLEMENTATION}

A Programming language Python is used to implement Graphic user interface (GUI). The Signals transmitted from the arduino received serially to personal computer using IEEE 802.4.15 receiver. The received signals are interfaced to the Personal computer for graphical analysis using Python programming language.

Internet of things application is used to retrieve the data using HTTP protocol through local area network. Thing speak is providing the facility of data logging from field instrumentation. The logged data is plotted using the python software.

This process unit is linked to the internet server with a separate I.P Address (192.168.1.49). The authorized person can access the process station at any location to observe the process parameters regulation mechanism.

\subsection{IMPLEMENTED ALGORITHM}

\section{Algorithm 1}

Step1:Initialize the PID error equation

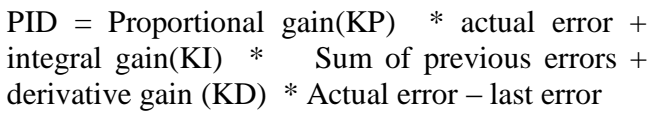

Step 2: Error $=$ Set point $($ SP $)-$ Process value $(P V)$

Step 3: Initialize the floating values for PID, SP,PV Initialize Error old $=\mathrm{SP}-\mathrm{PV}$

Step 4: Initialize

$$
\begin{aligned}
& \text { 'P' error }=\text { err } \\
& \text { 'I' error }=\text { Err old } \\
& \text { 'D' error }=\text { err-Err_old }
\end{aligned}
$$

Step 5: Initialize the Gain constants for $\mathrm{P}+\mathrm{I}+\mathrm{D}$

Equate KP with 0.1

Equate KI with 0.3

Equate KD with 0.02

Step 6: Initialize the dead zone

If

Err $<2$

And Err $\geq-2$

Then

Error $=$ ' 0 '

Else

Error is a constant variable

Step 7: Go to algorithm 2

\section{Algorithm 2}

Step 1: Initialize the ports

Sensors initialization

Port $\mathrm{C}_{-} 0$ is equated with temp sensor

Port $\mathrm{C}$ 1 is equated with flow sensor

Output device initialization

Port C_2 is equated with triac

Port D_0 is equated with Wireless transmitter

Step 2: Initialize the sample time with $1 \mathrm{sec}$

Step 3: Call the algorithm 1

If the error value is $>2$ and $\leq-2$

Then Enable the triac

Else read the data from port C_0

Step 4: Transmit the data serially through Port D_0 
Step 5: Display the state of Set point (SP) regulation If The Measured value is regulating above the set point

Then

Enable the RED LEDs

Apply the corrective mechanism Go to step 6 If

The measured value is regulating below the set point

Then

Enable the BLUE LEDs

Apply the corrective mechanism Go to step 6 Else

Go to step 7 of algorithm 3

Step 6: Adjust the Tuning values of Proportional $(\mathrm{P})$ + Integral (I) + Derivative (D) gains Call the Algorithm 3

\section{Algorithm 3}

Step 1: Adjust the Set point value

Step2: Assign 'ZERO' to Derivative and Integral Levels.

Step3: Define the safe value for Max_ power

Step 4: Adjust the Proportional gain to Minimum

Step 5: Initialize the' PID' Tuning adjust value 'Tn' $=0.1$

If

The gain 'KP' is minimum

Then

$\mathrm{KP}+\mathrm{Tn}$

If

The Set point value is increasing / decreasing

Then

Measure the oscillations period

If

The Oscillations are $\geq 5 \%$ of SP

Then

$\mathrm{Tn}=\mathrm{Tn}+0.1$

Else

Consider the value is ultimate gain

Measure the Ultimate Period of oscillations (Tu)

Step 6: Readjust the $\mathrm{P}+\mathrm{I}+\mathrm{D}$ values in step 5 of algorithm 1

$\mathrm{KP}=0.60 *$ ultimate gain

$\mathrm{KI}=2$ / Ultimate period of oscillations ' $\mathrm{Tu}$ ' $\mathrm{KD}=\mathrm{Tu} / 8$

Step 7: Go to step 6 of algorithm 1

\section{RESULTS \& ANALYSIS}

The experiments are conducted for various set points to regulate the process parameter with the proposed methodology. The results obtained are favorable to meet the challenges of the industry. The process parameter is regulated at the desired value. The results obtained at the field instrumentation are favorably good and the communication with the web server is enabling us to provide multiple control stations for constant monitoring even on offsite. This Work is more flexible to improve the plant management levels. Fig 5.1 represents the data extracted from the field instrumentation. The temperature reading, flow value and the output signal of the controller (Error value). The 'ON' and 'OFF' state of the heater is depending on the error signal. The error signal causes to enable the heater to raise the temperature of the fluid. A 'Zero' error signal will cause to turn off the heater. Fig 5.2 represents the process unit linked to the institute web site. This enables the authorities to monitor the plant status at any location. Fig 5.3 and Fig 5.4 depicts the graphical view of the plant parameters w.r.t to time. Placing the cursor on the graph will indicate the parameter values at any time axis. The status of the heating device is also specified to identify the process parameter above and below the set point values.

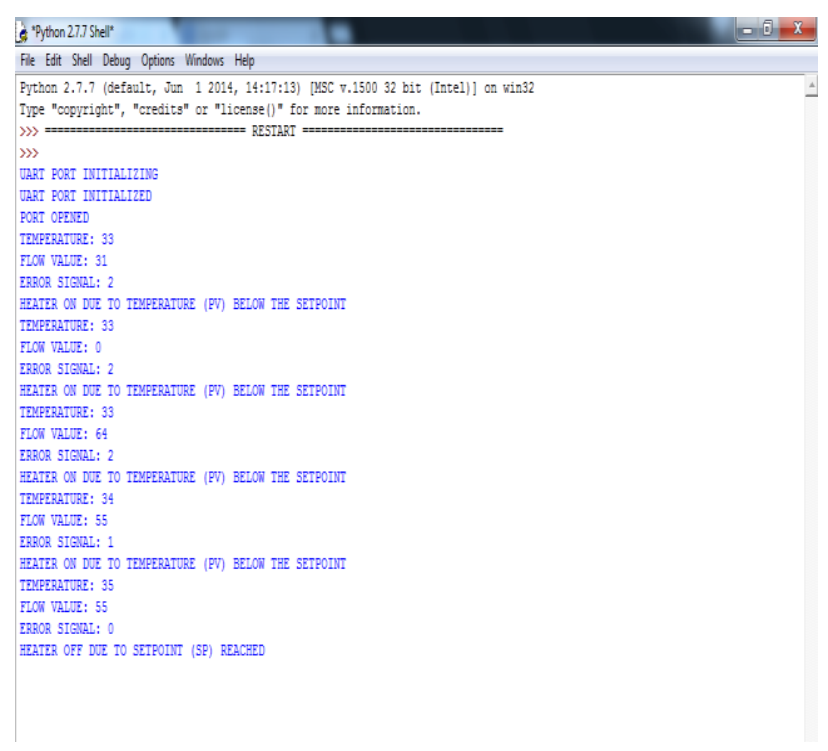

FIG 5.1 Data received at the control room

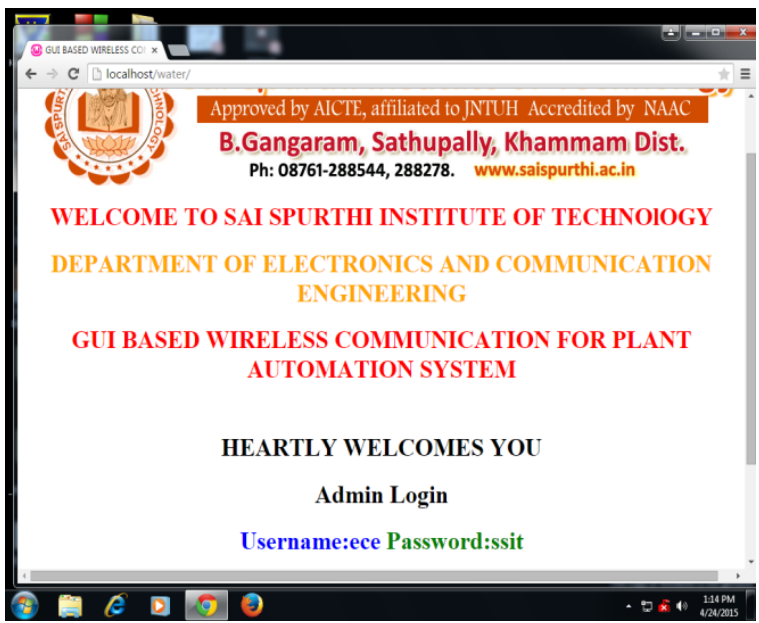

FIG 5.2 Title page linked to institute website

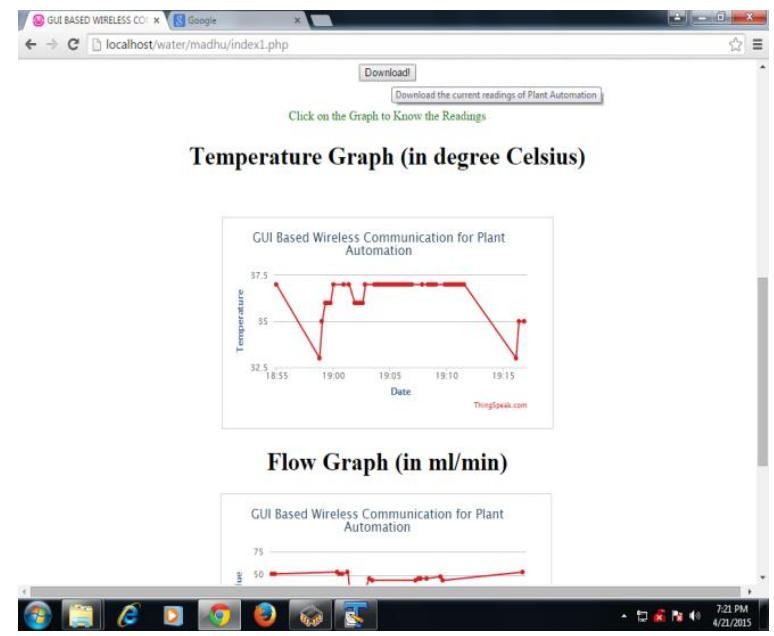

Fig 5.3. Graphical view of the process parametres 


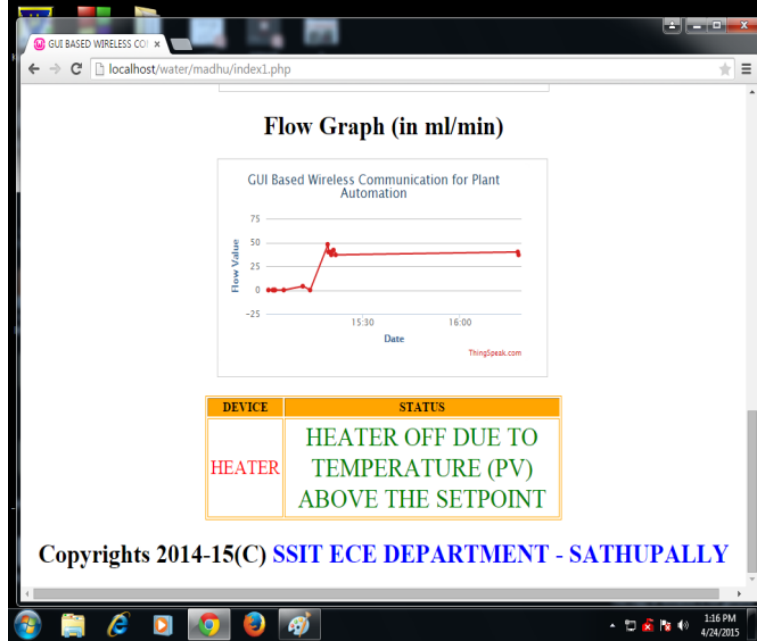

FIG 5.4 Status of the heating unit

\section{CONCLUSION}

Web Linked plant automation is the new era in process automation. The plant officer can supervise the state of the process parameters at any location.

The experiment conducted by considering two parameters. Python software enabled to for graphical analysis of the process parameters. The results obtained with this methodology are favorable to the expectations.

In future this work is proposed to be extended with more process parameters and back propagation neural networks in association with applied Fuzzy logic may improve the precision control of the plant parameters.

\section{ACKNOWLEDGMENTS}

The authors like to express sincere thanks to the management and principal of Sai Spurthi Institute of Technology for providing the fund and necessary infrastructure.

\section{REFERENCES}

[1] Mohammad Junaid Arshad, Amjad Farooq," GUI-Based Real-Time Industrial Automation Software ", Journal of American Science, Vol: 6(12), pages 96-101,2010.

[2] R. Kirubashankar et.al, "Design and implementation of web based remote supervisory control and information system" International Journal of Soft Computing and Engineering, Vol 1(4) : pages 43-51, Sep 2011.

[3] Salem Y. Al-Agtash, "PC-Based Automated Control System for Jordan Northern Grain Silo", Journal of Engineering, Vol 4 (12), 2012.

[4] Mr. Nilesh Yadav, Prof. Neeraj kuma, "A GUI (Graphical User Interface) framework For distribution automation through CAN (Control Area Network) Protocol", International Journal of Scientific \& Engineering Research, Vol 4 (6), June 2013.s

[5] Mohd Shariq khan et.al "Automated Optimization of process plant using Particle swarm optimization", proceedings of the $4^{\text {th }}$ international symposium on advanced control of industrial process, may 2011.

[6] Thoraya Obaid, et.al, "zigbee technology and its application in wireless home automation systems: a survey" , International Journal of Computer Networks \& Communications, Vol.6(4): 115-131, July 2014

[7] Engin Ozdemir, Mevlut Karacor, "Mobile phone based SCADA for industrial automation", ISA Transactions, Vol. 45(1): 67-75, January 2006.

\section{AUTHOR PROFILE}

Prof. K. Vidyasagar: Received B. Tech degree in Instrument Technology from Andhra University College of Engineering Visakhapatnam, M.E from P. S. G. Tech Coimbator. He is now a research scholar under the guidance of Dr. A. Bhujangarao, Andhra University. His current research interests include image processing in biomedical instrumentation and related embedded systems.

T. Chaitanya : Received B. Tech degree in Electronics and Communication Engineering from Bapatla Engineering College Bapatla, M. Tech Systems and Signal processing from Adams Engineering College, Paloncha. Her current research interests including VLSI Signal Processing and related embedded systems

D. Swapna: Received B. Tech degree in Electronics and Communication Engineering from Amaravathi University Maharashtra and $M$.Tech from Jagruthi Institute of technology Hyderabad. Her current research interest is embedded process system. 